\title{
Splenectomy during cytoreductive surgery in epithelial ovarian cancer
}

This article was published in the following Dove Press journal:

Cancer Management and Research

\author{
Hengzi Sun' \\ Xiaoning $\mathrm{Bi}^{\prime}$ \\ Dongyan Cao' \\ Jiaxin Yang' \\ Ming Wu' \\ Lingya Pan' \\ Huifang Huang' \\ Ge Chen ${ }^{2}$ \\ Keng Shen' \\ 'Department of Obstetrics and \\ Gynecology, Peking Union Medical \\ College Hospital, Chinese Academy \\ of Medical Sciences and Peking Union \\ Medical College, Beijing, China; \\ ${ }^{2}$ Department of General Surgery, \\ Peking Union Medical College \\ Hospital, Chinese Academy of Medical \\ Sciences and Peking Union Medical \\ College, Beijing, China
}

Correspondence: Dongyan Cao Department of Obstetrics and Gynecology, Peking Union Medical College Hospital, Chinese Academy of Medical Sciences and Peking Union Medical College, No. I ShuaiFuYuan, Dongcheng District, Beijing 100730, China

Tel +861069156114

Fax +861068419898

Email caodongyan@pumch.cn
Background: The aim of the study was to analyze the underlying causes and application of splenectomy in patients with epithelial ovarian cancer (EOC) and assess its effect on the surgical satisfaction and prognosis of these patients.

Materials and methods: Clinical data of patients with ovarian epithelial cancer treated with cytoreductive surgery were collected from 2000 to 2015 at Peking Union Medical College Hospital. Results: A total of 2,882 patients underwent ovarian cancer cytoreductive surgery at Peking Union Medical College Hospital between 2000 and 2015, of whom 38 (1.3\%) also underwent spleen resections. Of these 38 patients, one underwent splenectomy due to intraoperative trauma, whereas the remaining 37 patients underwent splenectomy due to splenic metastasis. Among these 37 patients, 27 underwent resection due to direct tumor spread in the spleen and 10 underwent resection due to hematogenous metastasis. For subsequent first-line chemotherapy, 22 patients were platinum sensitive and 15 were platinum resistant. Overall median survival and the postsplenectomy median survival time were 106 and 75 months, respectively. The overall median survival in secondary cytoreduction was 101 months compared with 20.3-56 months in literature reviews. Univariate analysis revealed that platinum resistance to first-line chemotherapy, suboptimal surgery, and hematogenous metastasis influenced survival. Chemosensitivity and residual disease were identified as independent risk factors by multivariate analysis. We also report a literature review concerning the efficacy and safety of splenectomy during cytoreductive surgery in EOC. Conclusion: Approximately $1.3 \%$ of patients with EOC underwent spleen resection during initial cytoreductive surgery and more often during recytoreductive surgery. Tumor involvement was the most common indication for splenectomy, and rare patients underwent splenectomy due to intraoperative trauma. Most patients achieved optimal surgery, and thus their overall survival and postsplenectomy survival rates were longer. The prognosis of patients was closely related to chemosensitivity and presence of residual tumors. Splenectomy should be attempted in all patients with splenic involvement in whom optimal cytoreductive surgery was achievable, no matter in primary or secondary cytoreduction.

Keywords: optimal debulking surgery, chemosensitivity, postoperative complication, splenic involvement, metastasis, prognosis

\section{Introduction}

Ovarian cancer has the highest mortality rate of all gynecologic cancers, because its early symptoms are nonspecific, and most patients present an advanced stage of this disease during initial diagnosis. ${ }^{1}$ In total, 52,100 new cases and 22,500 deaths are estimated to have occurred in China in 2016, representing the second highest cause of gynecologic cancer-related deaths. ${ }^{2}$ 
Cytoreductive surgery followed by combination platinumbased chemotherapy has long been considered the appropriate treatment for patients with advanced epithelial ovarian cancer (EOC). Prospective clinical trials and retrospective studies have revealed that optimal cytoreduction improves survival and that postoperative residual disease is one of the most important prognostic parameters. ${ }^{3,4}$ Maximal surgical effort is a feasible and effective therapeutic intervention. However, due to technical reasons, as well as many other objective factors, optimal surgical resection is not always achieved because it may require extensive and complicated procedures to resect distant metastases, such as liver or pancreatic resection. ${ }^{5}$

According to the 2016 National Comprehensive Cancer Network guidelines, to achieve optimal surgical cytoreduction (in all stages) of epithelial ovarian carcinoma, we may consider the removal of relevant abdominal organs such as radical pelvic dissection, bowel resection and/or appendectomy, diaphragm resection or other peritoneal surface stripping, partial hepatectomy, partial gastrectomy, partial cystectomy and/or ureteroneocystostomy, cholecystectomy, and/or distal pancreatectomy, including, splenectomy. ${ }^{6}$ However, the clinical practice of splenectomy in EOC cytoreductive surgery is rare.

Therefore, we performed a retrospective analysis of the underlying causes and application of splenectomy during cytoreductive surgery in patients with epithelial ovarian carcinoma (EOC). We also assessed the effect of splenectomy on the prognosis and surgical satisfaction of these patients.

\section{Materials and methods}

After institutional review board approval, we retrospectively reviewed the data of patients who underwent splenectomy at the time of cytoreductive surgery for EOC at the Department of Obstetrics and Gynecology, Peking Union Medical College Hospital, Chinese Academy of Medicine Sciences, Beijing, between January 2000 and December 2015. Inclusion criterion was the diagnosis of EOC with splenic involvement confirmed by histopathological analysis; exclusion criterion was splenectomy due to nontumor factors, such as intraoperative trauma.

Data collected included age at diagnosis of EOC, all perioperative details including the cytoreductive surgery times of splenectomy performed, stage, histopathological subtype, degree of differentiation, residual tumor, type of splenic involvement (implantation or hematogenous metastasis), reason for splenectomy, adjuvant therapy, and follow-up data. Disease stage was defined according to the 2014 International Federation of Gynecology and Obstetrics ovarian cancer staging guidelines. Residual tumor was defined according to the maximal dimension of the single largest cancer nodule at the end of cytoreductive surgery with synchronous splenectomy. Optimal cytoreductive surgery of EOC was defined as $<1 \mathrm{~cm}$ residual tumor volume.

A comprehensive search of the MEDLINE/PubMed database was performed for articles published from January 2000 to December 2017. The following keywords were used: "splenectomy" and "ovarian cancer". Studies were selected for original reports on the outcome of splenectomies during cytoreductive surgery in EOC. Case reports were excluded.

The differences between different subgroups were analyzed by the log-rank test. Survival curves were generated using Kaplan-Meier method. Statistics and graphics were performed using the GraphPad prism 5.00 program.

\section{Results}

From 2000 to 2015, 2,882 patients underwent EOC cytoreductive surgery at Peking Union Medical College Hospital, of whom only 38 patients $(1.3 \%)$ underwent spleen resection. Of these 38 patients, $7(18.4 \%)$ and $31(81.6 \%)$ underwent splenectomy during primary cytoreduction and recytoreduction, respectively. Of the 31 patients who underwent splenectomy during recytoreduction, 24 patients underwent splenectomy during secondary cytoreduction, six during tertiary cytoreduction, and one patient was beyond tertiary treatment. Table 1 summarizes the characteristic of all patients, including, age, disease stage, tumor grade, and residual disease status after cytoreductive surgery.

The median age of the 38 enrolled patients was 53 years (range, 34-75 years). The distribution of surgical stage was as follows: $2(5 \%)$ stage I-II patients, 27 (71\%) stage III patients, and 9 (24\%) stage IV patients. Among all patients, 28 patients had serous carcinoma, four had endometrioid carcinoma, three had clear cell carcinoma, and three had Müllerian ductal carcinoma.

Tumor involvement was the most common indication for splenectomy. Only one patient underwent splenectomy due to intraoperative trauma to the spleen with bleeding that could not be managed by usual hemostatic techniques; the remaining 37 patients $(97.4 \%)$ required splenectomy for direct splenic involvement by tumor. However, the route of splenic involvement was variable. Twenty-seven patients (73\%) underwent splenectomy due to direct tumor spread/implantation on the spleen, including 21 cases with multiple lesions in the upper abdomen, such as peritoneal or/and mesentery lesions; six cases had isolated lesions in the splenic hilum and/or capsule. Ten patients (27\%) underwent splenectomy due to hematogenous metastasis, including four cases with 
only splenic parenchymal metastases and six cases with other intraperitoneal (IP) lesions. The case in which splenectomy was performed due to intraoperative trauma, which could not be used for final statistical analyses, was formally excluded. Thus, the final data included 37 cases.

Although optimal cytoreductive surgery was attempted in all cases, it was only achieved in $34(91.9 \%)$ cases. In the other three cases, tumor involvement of the right cardiophrenic angle lymph node, porta hepatis, or diaphragm was diagnosed intraoperatively. For subsequent first-line chemotherapy, $22(59 \%)$ patients were platinum sensitive, which means that $59 \%$ of patients had no relapse within 6 months of withdrawal after remission. Fifteen (41\%) patients were platinum resistant, meaning that all these patients relapsed

Table I Characteristics of patients

\begin{tabular}{|c|c|c|}
\hline Characteristics & No. & $\%$ \\
\hline Age (years), median (range) & $53(34-75)$ & \\
\hline \multicolumn{3}{|l|}{ Cytoreductive surgery } \\
\hline Primary & 7 & 18.4 \\
\hline Secondary & 24 & 63.2 \\
\hline Tertiary & 6 & 15.8 \\
\hline Beyond tertiary cytoreduction & I & 2.6 \\
\hline \multicolumn{3}{|l|}{ Stage } \\
\hline I-II & 2 & 5.3 \\
\hline III & 27 & 71.0 \\
\hline IV & 9 & 23.7 \\
\hline \multicolumn{3}{|l|}{ Histological subtype } \\
\hline Serous & 28 & 73.7 \\
\hline Endometrioid & 4 & 10.5 \\
\hline Clear cell & 3 & 7.9 \\
\hline Müllerian ductal & 3 & 7.9 \\
\hline \multicolumn{3}{|l|}{ Differentiation grade } \\
\hline GI & 9 & 26.3 \\
\hline G2 & 10 & 26.3 \\
\hline G3 & 14 & 31.6 \\
\hline Unknown & 5 & 15.8 \\
\hline \multicolumn{3}{|l|}{ Reason for splenectomy } \\
\hline Achieve optimal cytoreduction & 37 & 97.4 \\
\hline Intraoperative trauma & 1 & 2.6 \\
\hline \multicolumn{3}{|l|}{ Complications } \\
\hline Thromboembolic & I & 2.6 \\
\hline Pulmonary infection & 2 & 5.2 \\
\hline \multicolumn{3}{|l|}{ Residual disease $^{a}$} \\
\hline Optimal & 34 & 91.9 \\
\hline Suboptimal & 3 & 8.1 \\
\hline \multicolumn{3}{|l|}{ Type of splenic involvement ${ }^{a}$} \\
\hline Implantation metastasis & 27 & 73 \\
\hline Hematogenous metastasis & 10 & 27 \\
\hline \multicolumn{3}{|l|}{ The sensitive to first-line } \\
\hline \multicolumn{3}{|l|}{ chemotherapy ${ }^{\mathrm{a}}$} \\
\hline Platinum sensitive & 22 & 59.5 \\
\hline Platinum resistance & 15 & 40.5 \\
\hline
\end{tabular}

Note: ${ }^{a}$ Cases in which splenectomy was performed due to intraoperative trauma were excluded. within 6 months of remission. Among the 37 patients, one $(2.6 \%)$ had a postoperative complication of thromboembolic events, whereas two patients had pulmonary infection.

Median survival was 106 months, with estimated 3-, 5-, and 10 -year survival rates of $87 \%, 76 \%$, and $27 \%$, respectively. All patients had a postsplenectomy median survival time of 45 months, with estimated 3- and 5-year survival rates of $59 \%$ and $42 \%$, respectively (Figure 1). The overall median survival in secondary cytoreduction was 101 months, and the time of media follow-up in primary cytoreduction was 20 months (7-29 months).

We compared the outcomes between patients with splenic involvement due to implanted metastasis vs hematogenous metastasis. The median overall survival for patients with implanted metastasis was 101 months, with a 5-year survival rate of $82 \%$, whereas it was 68 months for patients with hematogenous metastasis, with a 5-year survival rate of $57 \%(P=0.76)$. The postsplenectomy median survival for patients with implanted metastases was 45 months, with a 5 -year postsplenectomy survival rate of $47 \%$, whereas it was 26 months for patients with hematogenous metastasis, with an expected 5-year postsplenectomy survival rate of $22 \%$ $(P=0.072)$ (Figure 2$)$. The postsplenectomy survival was influenced by the type of splenic involvement, but did not reach statistical significance.

We also compared the outcomes between patients with platinum-sensitive tumors vs those with platinum-resistant tumors. A significantly improved survival rate was obtained for patients with platinum-sensitive disease. Patients with platinum-sensitive and platinum-resistance tumors had an overall median survival of 111 months and 68 months and an estimated 5-year survival rates of $89 \%$ and $61 \%$, respectively $(P=0.004)$. The postsplenectomy median survival

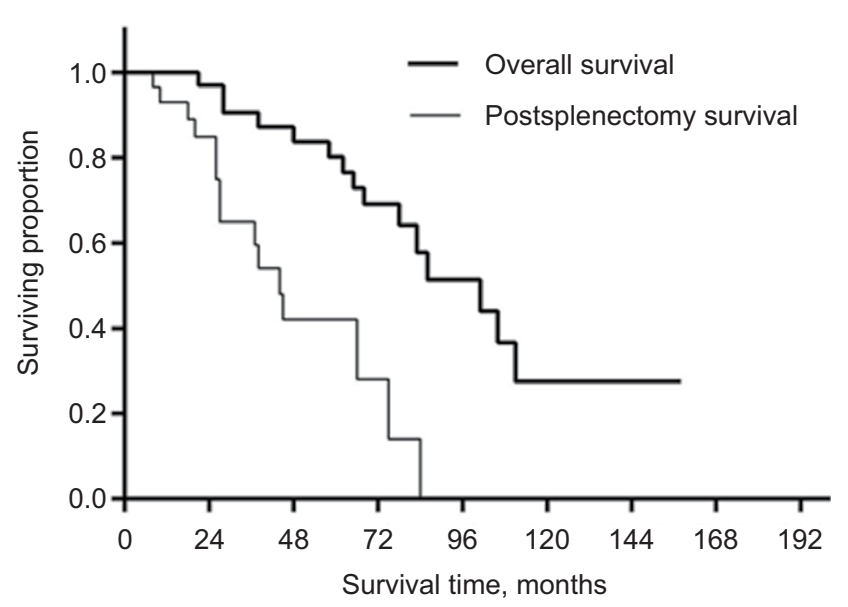

Figure I The overall survival and postsplenectomy survival by splenectomy. 
rates were 75 months and 27 months, with expected 5-year postsplenectomy survival rates of $52 \%$ and $29 \%$, respectively $(P=0.047)$ (Figure 3).

We attempted to compare the outcomes in patients with varied residual disease after cytoreductive surgery; however, the statistical power was very low as only $3(8 \%)$ cases were suboptimal. Patients with optimal and suboptimal cytoreductive surgery had an overall median survival of 101 and 86 months, respectively $(P=0.392)$; the postsplenectomy median survival rates were 45 and 23 months, respectively $(P=0.066)$ (Figure 4).

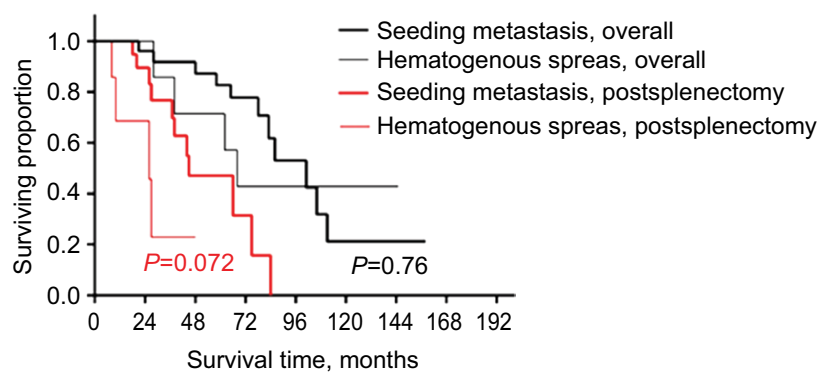

Figure 2 The overall survival and postsplenectomy survival by splenectomy of the cases of splenic involvement presenting implanted metastasis vs hematogenous metastasis.

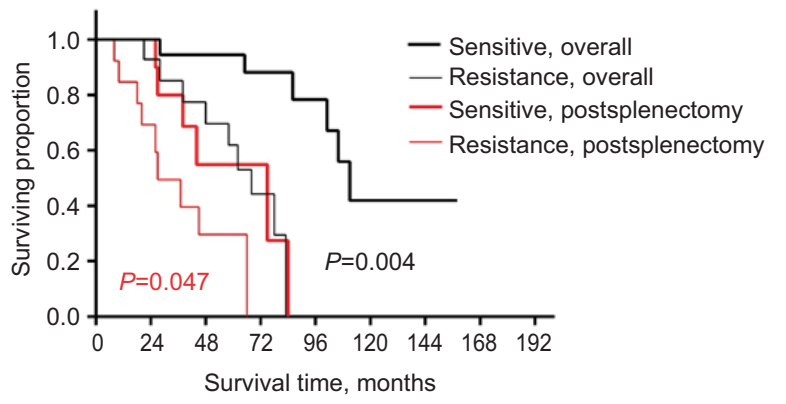

Figure 3 The overall survival and postsplenectomy survival by splenectomy of the cases with platinum sensitivity vs platinum resistance.

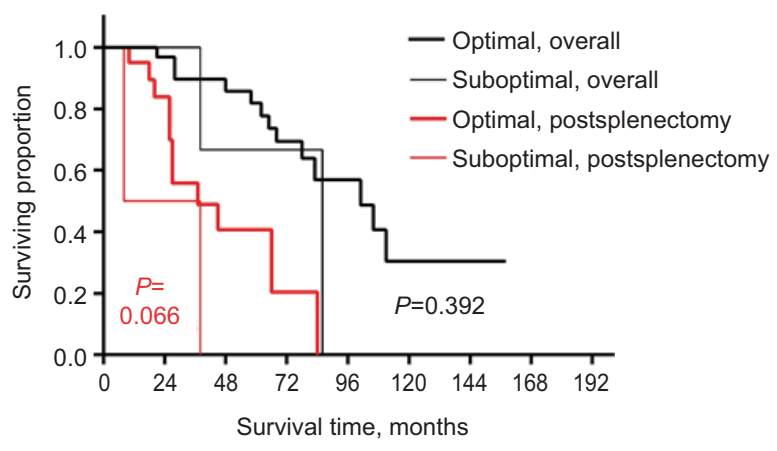

Figure 4 The overall survival and postsplenectomy survival by splenectomy of the cohort of patients who underwent suboptimal and optimal cytoreduction.
The correlation between age ( $<50$ or $>50$ years), tumor grade, histological subtype, stage, residual disease, type of splenic involvement, sensitivity to first-line chemotherapy, and prognosis was analyzed. Univariate analysis revealed that sensitivity to first-line chemotherapy, residual disease, and the type of splenic involvement influenced survival. Chemosensitivity and residual disease were identified as independent risk factors by multivariate analysis (Table 2).

A comprehensive review on splenectomy during cytoreductive surgery in EOC was implemented. Data from 10 original studies involving splenectomy along with the present study were incorporated (Table 3). About 412 cases underwent splenectomy as a part of cytoreductive surgery in EOC, most of which were FIGO III-IV and serous carcinoma. Of the 412 cases, $77 \%$ patients underwent splenectomy during primary cytoreduction, of which $85 \%$ patients achieved optimal cytoreduction. However, only $33 \%-70 \%$ patients achieved optimal cytoreduction in secondary cytoreduction. The median survival was 21.6-58.4 months and 20.3-56 months in primary and secondary cytoreduction, respectively. The major complications were thrombocytosis, embolism, fever, and sepsis. Besides, independent risk factors were variance in each report. Residual disease status significantly correlated to overall survival at cytoreductive surgery with splenectomy in EOC.

\section{Discussion}

Over $60 \%$ of patients with EOC at the time of diagnosis present an advanced stage of this disease. The 2016 NCCN guidelines suggested that all tumor lesion should be removed as much of the malignant tissue as possible in advanced ovarian cancer, even the lesion involved in other organ, to achieve the optimal cytoreductive surgery, and the efficacy of complete cytoreduction has been widely accepted and demonstrated by large series of cases.

However, splenectomy as a part of cytoreductive surgery in EOC demonstrating splenic metastasis is rarely seen in clinical practice. ${ }^{7,8}$ There are two reasons for this

Table 2 Multivariate analysis of postsplenectomy survival in cytoreductive surgery $(P=0.01)$

\begin{tabular}{llll}
\hline Covariate & Risk & $\mathbf{9 5 \%} \mathbf{C l}$ & $\mathbf{P}$ \\
\hline $\begin{array}{l}\text { First-line chemotherapy } \\
\quad \text { Sensitive }\end{array}$ & $\mathrm{I}$ & & \\
$\quad$ Resistance & 4.73 & $1.19-18.7$ & 0.027 \\
$\begin{array}{l}\text { Residual disease } \\
\text { Optimal }\end{array}$ & & & \\
$\quad$ Suboptimal & $\mathrm{I}$ & & \\
\hline
\end{tabular}




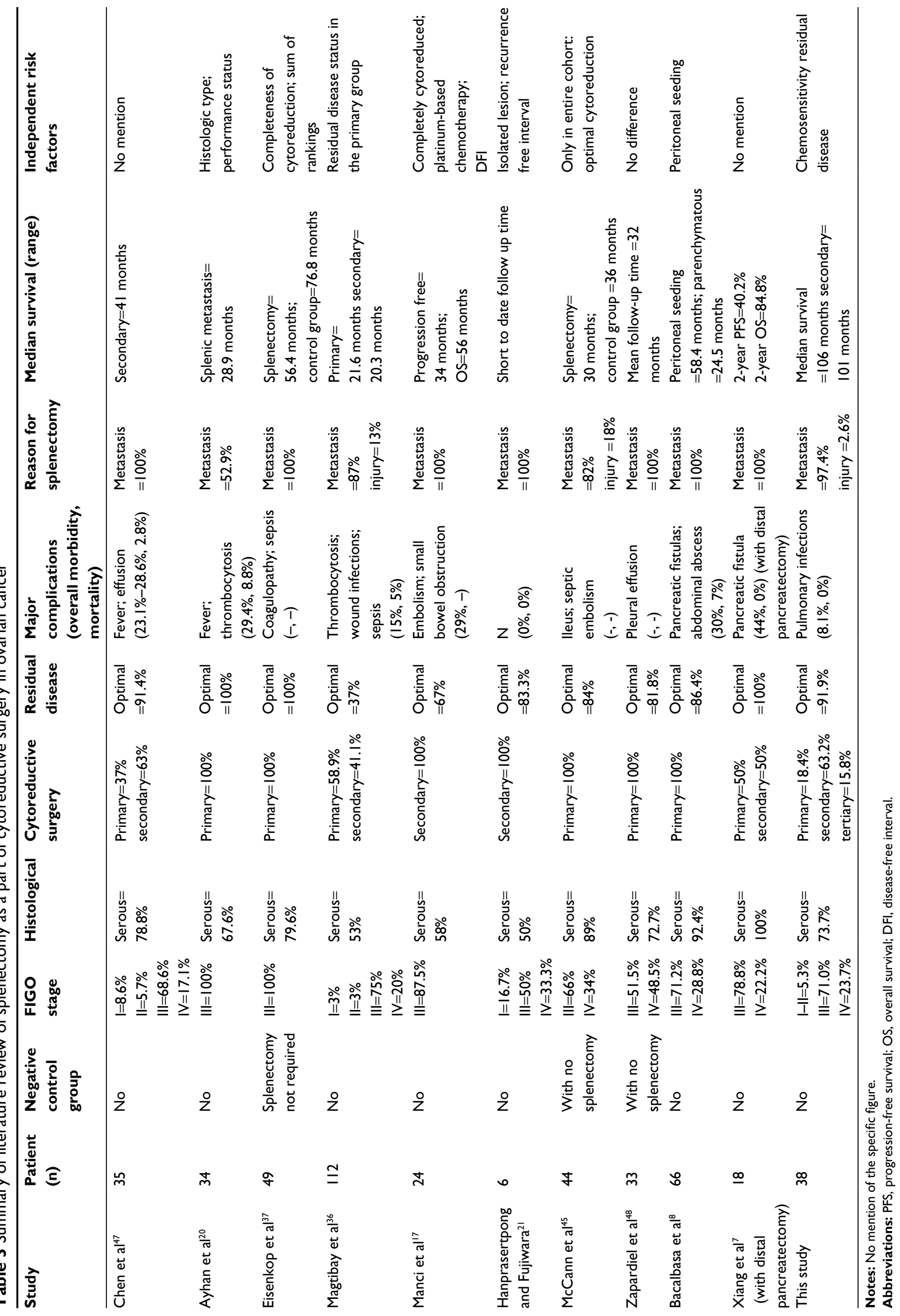


phenomenon. First, many studies suggest that the splenic capsule could act as a shield and its contractile properties might also be involved by squeezing the tumor cells out. Moreover, the spleen is an immune organ with a population of lymphoid tissue and lymphocytes that can kill invading tumor cells. The tortuosity of the splenic artery and abdominal fluid circulation could also be responsible for the low rate of splenic involvement. ${ }^{9-11}$ Peters et $\mathrm{al}^{12}$ proposed a hypothesis that the spleen is highly active in terms of physiological granulocyte destruction, which inhibits the survival of cancer cell in the spleen owing to proapoptotic signals. However, according to the advanced Phase III global study, spleen is a lymphoid organ rich in CD8 (cytotoxic T-cell) trafficking the perifollicular area, thus playing a major role in immune surveillance and immune editing process in oncogenesis. ${ }^{13}$ Second, splenectomy is generally not performed in cases presenting advanced disease where debulking would be suboptimal or large tumor masses appear to be unresectable. In our study, $\sim 1.3 \%$ of patients with ovarian cancer underwent spleen resection during cytoreductive surgery, which was lower than the literatures. We considered the reason was various. First, splenic metastasis occurs rarely in malignant tumors with the prevalence ranging from $2.3 \%$ to $7.1 \%{ }^{9}$ The exact incidence of splenic metastasis from ovarian cancer is hard to determine. Metastatic EOC to the spleen is an uncommon clinical event, although one autopsy study reported that splenic metastasis was found in $20 \%$ ovarian cancer patients. ${ }^{14}$ Second, it may be due to the fact that our data are based on the Chinese population. In the past 15 years, a total of $\sim 3,000$ cases of EOC have undergone cytoreductive surgery, which may be statistically different from studies abroad. Data from another related study in China in 2016 showed that 91 (2.2\%) of the 4,100 patients with EOC received splenectomy, which was consistent with our study. ${ }^{7}$ Third, in our study, comprehensive evaluation of risks and benefits before surgery should be conducted for ovarian cancer patients with splenic metastasis. Only those patients who possibility achieved optimal cytoreduction would underwent splenectomy. Besides, the frequency of splenectomy, which focused on attempting cytoreduction to consistently less than $5 \mathrm{~mm}$, has increased during the past half decades. We also found that, among patients who were treated with splenectomy, approximately $82 \%$ underwent this procedure during re-cytoreductive surgery. However, literature reviews revealed that most patients underwent splenectomy during primary cytoreduction. We considered it was due to the fact that the true hematopoietic metastasis of splenic parenchyma at the initial treatment of EOC is uncommon. For our center, as long as the primary cytoreduction can achieve optimal debulking, we usually take only the resection of omental instead of the spleen in the case with omental metastasis near the splenic portal to reduce surgical trauma. Second, Peking Union Medical College Hospital is a national referral hospital; many patients have underwent primary cytoreduction in local hospital and then were referred to our center after recurrence. In many hospitals, the primary debulking surgery may be performed by nongynecologic oncologists or lack the help of surgeons and the technical conditions for splenectomy. Lv et a $1^{15}$ reported that splenic metastasis of ovarian carcinoma largely occurs postoperatively, following subsequent surgeries or years after radiotherapy and chemotherapy. Splenic metastasis is primarily accompanied by dissemination to the omentum majus and pelvic cavity. Isolated metastatic splenic lesions only occur in rare cases. This may be related to chemotherapy, which can inhibit the normal immune function even while extending survival. ${ }^{16}$ Manei et al ${ }^{17}$ reported that patients with splenic metastasis most often present with poorly differentiated serous adenocarcinomas - a pathological ovarian cancer subtype - which is highly invasive. Our data also show that serous adenocarcinomas accounted for approximately $74 \%$, while approximately $70 \%$ of patients had poorly differentiated-type tumors.

Main reasons for splenectomy in ovarian cancer include splenic metastasis, perisplenic tumor infiltration, and intraoperative trauma..$^{18}$ However, with improvement of surgical techniques, the occurrence of intraoperative trauma is minimal. Only one patient underwent splenectomy due to intraoperative trauma in our study. Advanced ovarian cancer involves the abdomen in most cases, and the spleen is usually involved as part of vast upper abdominal disease spread or, less frequently, as isolated disease recurrence. ${ }^{19,20}$ Metastatic splenic lesions are always more than $1 \mathrm{~cm}$ and it is difficult to achieve optimal cytoreductive surgery for those patients without splenectomy, which may influence prognosis. ${ }^{21}$

In the present study, 34 of 37 patients who underwent splenectomy achieved optimal cytoreductive surgery. Overall median survival and postsplenectomy median survival were 106 and 45 months, respectively, which was significantly better than survival time of patients reported by previous studies. This was mainly because most cases were adequately evaluated, leading to early general surgical and Intensive Care Unit (ICU) support. For ovarian cancer patients who conducted splenectomy to achieve optimal cytoreduction after evaluation, we will contact the ICU in advance to prepare for major bleeding and other organ injuries that may occur during surgery, so that we can monitor vital signs closely after surgery to prevent and treat 
postoperative complications as soon as possible. Therefore, those patients with high risk of postoperative complications should benefit more from ICU support. Additionally, standard and adequate chemotherapy was required, Previous randomized trials and other studies reported that IP/intravenous (IP/ IV) chemotherapy also has been shown to have a survival advantage compared with IV chemotherapy alone in patients with stage III EOC who have undergone optimal primary cytoreductive surgery. ${ }^{22-25}$ However, in light of latest studies, IP therapy after neoadjuvant chemotherapy and interval debulking surgery or optimally surgical with bevacizumab may not have the same degree of survival advantage over IV therapy. ${ }^{26,27} \mathrm{In}$ addition, when choosing the type of adjuvant treatment, doctors and patients should consider convenience, potential toxicities, quality of life, and the possible survival benefit. ${ }^{28} \mathrm{We}$ also find that overall survival in secondary cytoreduction was better (101 months) compared with 20.3-56 months in previous studies. We believe that it was due to the optimal cytoreductive surgery in most patients with secondary cytoreduction. However, there are limitations in trying to assess the outcomes of this procedure in primary cytoreduction, which was due to the short to-date follow-up time (10-29 months). Thus, splenectomy should be attempted in all patients with splenic involvement in whom optimal cytoreductive surgery was achievable, no matter in primary or secondary cytoreduction. Patients with unresectable massive peritoneal involvement as well as with a miliary pattern of distribution, wide spread diaphragmatic disease infiltrating carcinosis or confluent nodules to the most part of the diaphragmatic surface, large mesenteric infiltrating nodules, or an involvement of the root of the mesentery were supposed on the basis of limited movements of the various intestinal segments. Tumor diffusion was observed along the omentum up to the large stomach curvature; a bowel resection was assumed or when miliary carcinosis on the ansae was observed, an obvious neoplastic involvement of the gastric wall was observed; any liver surface lesions were considered the difficulties factor of surgical debulking. ${ }^{29}$ In our study, tumor involvement of the right cardiophrenic angle lymph node, porta hepatis, or diaphragm was diagnosed intraoperatively and failed to achieve optimal cytoreduction finally. Therefore, the possibility of optimal cytoreductive surgery should be adequately assessed before splenectomy, especially in determining whether the hilar mass or diaphragm region could be removed.

According to literatures, the major postsplenectomy complications included abdominal complications such as intraabdominal collections, abscesses, anastomotic leaks, and pancreatic fistulas; pulmonary complications such as pleural effusion, pneumonia, and pulmonary embolism; infectious complications such as anastomotic leaks or abscesses, septic, and overwhelming postsplenectomy infection (OPSI); and hematologic complications such as deep-vein thrombophlebitis and portal system thrombosis. Recently, ${ }^{30-34}$ Dagbert et al ${ }^{35}$ evaluated the impact of splenectomy during cytoreductive surgery and hyperthermic intraperitoneal chemotherapy (CRS+HIPEC) on postoperative outcomes; the most common abdominal complications in the splenectomy group were left upper quadrant abscess, anastomotic leaks, and pancreatic fistulas, and the most common pulmonary complications were pleural effusions, pulmonary embolisms, and pneumonia. The number of pulmonary complications was significantly greater in the splenectomy group. Major complications of splenectomy include postoperative bleeding, infection, thrombocytosis, thrombosis, and gastric and pancreatic tail injury. Magtibay et $\mathrm{al}^{36}$ reported that among 112 patients who underwent splenectomy, the overall complication rate was $23 \%(26 / 112)$. Seven $(6.3 \%)$ had wound infections, five $(4.5 \%)$ had postoperative pneumonia, nine $(8 \%)$ had thromboembolic events, and five (4.5\%) had sepsis; the authors suggested that when performed as part of cytoreductive procedures in patients with EOC, splenectomy was associated with modest but acceptable morbidity and mortality rates. The extent of surgical tumor cytoreduction in patients with EOC should be determined on a case-by-case basis. However, others report that the risk of early complications, such as pancreatic fistula secondary to distal pancreatectomy due to confluent disease adherent to the splenic hilum and pancreas, can be reduced ${ }^{17}$ Additionally, long-term infectious complications of splenectomy were effectively prevented with appropriate vaccination. ${ }^{37}$ The elevated risk of infection is culminated in the first 2 years after patients underwent splenectomy and last through lifetime. Streptococcus pneumoniae is the major pathogen in postsplenectomy sepsis, accounting for $50 \%$ $90 \%$ of all infections. ${ }^{38}$ OPSIs are rare but well-described, life-threatening events that can occur after splenectomy. The potential risk of infections of S. pneumoniae, Neisseria meningitidis, and Haemophilus influenzae became maximal with the extreme gravity OPSI in splenectomized patient. Waghorn ${ }^{39}$ determined, however, that only $31 \%$ of patients who had an OPSI had previously received the appropriate pneumococcal vaccine. When elective splenectomy is planned, it is preferable to administer the vaccine at least 2 weeks before surgery to ensure better immunogenicity. For emergency splenectomy or when vaccines were not administered beforehand, the immunizations were recommended 
to be administered at least 2 weeks after surgery because the vaccine response is lower in the first 2 weeks after splenectomy. ${ }^{40}$ The incidence of complications after splenectomy in patients with ovarian cancer was not significantly different from that of patients who did not undergo splenectomy. ${ }^{7}$ According to latest literatures, the overall morbidity and mortality ranged from $0 \%$ to $30 \%$, and $0 \%$ to $8.8 \%$, respectively (Table 3 ). In our study of 37 patients, only one $(2.6 \%)$ had a postsplenectomy-related complication consisting of thromboembolic events, whereas two patients developed pulmonary infections and the morbidity and mortality were $8.1 \%$ and $0 \%$, respectively. We considered that this low rate of complications was due to accurate patient assessment and effective prevention by antibiotic prophylaxis and other strict nursing management.

There are rare reports concerning prognostic factors in patients with ovarian cancer with splenic metastasis. Spencer et $\mathrm{al}^{41}$ suggested that parenchymal lesions (a more common feature of relapsed disease state) were significantly less likely to respond to treatment than surface lesions. Another study reported an association between metastatic splenic tumor and poor overall prognosis in patients with widespread visceral dissemination of primary tumor. ${ }^{16}$ Solitary splenic metastases, which is usually indicative of hematogenous spread, was reported to present more moderate disease with better prognosis. ${ }^{42}$ Kugimiya et $\mathrm{al}^{43}$ reported that good prognosis may be achieved with chemotherapy after splenectomy in solitary splenic metastasis. Moreover, Marriqoun-Haarris et $\mathrm{al}^{44}$ reported good prognosis in a case of isolated recurrent splenic ovarian cancer treated with surgery and no adjuvant therapy. Recent research shows that the presence of parenchymal splenic metastases during primary cytoreduction for advanced stage ovarian cancer was associated with significantly poorer survival when compared to hilar or peritoneal seeding. ${ }^{19}$ Due to the limited number of cases presenting splenic involvement and the various models of involvement (peritoneal, parenchymatous or even lymphatic seeding), initial studies reported conflicting results, failing to demonstrate if the presence of splenic involvement is itself a poor prognosis factor. ${ }^{20,37,45,46}$ However, Splenic hilum metastasis of EOC has been considered an obstacle to optimal cytoreduction in most institutes, because researchers believe that spleen metastasis increased the difficulty to achieve optimal cytoreductive surgery. ${ }^{7}$ In the present study, although post-splenectomy survival was influenced by the type of splenic involvement, this effect was not statistical significant. We considered that this could be due to the small patient number in our study and the prognostic factors need more clinical observation. Sensitivity to first-line platinum-based chemotherapy was identified as an independent risk factor on multivariate analysis. Therefore, maximal survival benefit occurred in the setting of optimal cytoreductive surgery and chemosensitivity of the patients with advanced EOC.

\section{Conclusion}

In conclusion, $\sim 1.3 \%$ of patients with ovarian cancer underwent spleen resection during cytoreductive surgery, and most often during recytoreductive surgery. Tumor involvement was the most common indication for splenectomy and rare patients underwent splenectomy due to intraoperative trauma to the spleen with bleeding that could not be managed by usual hemostatic techniques. Most patients achieved satisfactory pelvic cavity tumor reduction, and thus their overall survival and postsplenectomy survival rates were longer. Patient prognosis was closely correlated with chemosensitivity and the presence of residual tumor. Splenectomy should be attempted in all patients with splenic involvement in whom optimal cytoreductive surgery was achievable, no matter in primary or secondary cytoreduction.

\section{Acknowledgments}

This study was funded by the National Natural Science Foundation of China (81572576 [Shen Keng]) and CAMS Initiative for Innovative Medicine (CAMS-2017-12M-1-002 [Keng Shen]).

\section{Disclosure}

The author reports no conflicts of interest in this work.

\section{References}

1. Karam AK, Santillan A, Bristow RE, et al. Tertiary cytoreductive surgery in recurrent ovarian cancer: selection criteria and survival outcome. Gynecol Oncol. 2007;104(2):377-380.

2. Chen W, Zheng R, Baade PD, et al. Cancer statistics in China, 2015. CA Cancer J Clin. 2016;66(2):115-132.

3. Ibeanu OA, Bristow RE. Predicting the outcome of cytoreductive surgery for advanced ovarian cancer: a review. Int J Gynecol Cancer. 2010;20(Suppl 1):S1-S11.

4. Marchetti C, Pisano C, Facchini G, et al. First-line treatment of advanced ovarian cancer: current research and perspectives. Expert Rev Anticancer Ther. 2010;10(1):47-60.

5. Shih KK, Chi DS. Maximal cytoreductive effort in epithelial ovarian cancer surgery. J Gynecol Oncol. 2010;21(2):75-80.

6. Morgan RJ, Jr., Armstrong DK, Alvarez RD, et al. Ovarian Cancer, Version 1.2016, NCCN Clinical Practice Guidelines in Oncology. JNCCN. 2016;14(9):1134-1163.

7. Xiang L, Tu Y, He T, et al. Distal pancreatectomy with splenectomy for the management of splenic hilum metastasis in cytoreductive surgery of epithelial ovarian cancer. J Gynecol Oncol. 2016;27(6):e62.

8. Bacalbasa N, Balescu I, Dima S, Brasoveanu V, Popescu I. Splenectomy as part of cytoreductive surgery in recurrent epithelial ovarian cancer. Anticancer Res. 2015;35(9):5097-5101.

9. Koh YS, Kim JC, Cho CK. Splenectomy for solitary splenic metastasis of ovarian cancer. BMC Cancer. 2004;4:96. 
10. Otrock ZK, Seoud MA, Khalifeh MJ, Makarem JA, Shamseddine AI. Laparoscopic splenectomy for isolated parenchymal splenic metastasis of ovarian cancer. Int J Gynecol Cancer. 2006;16(5):1933-1935.

11. Alloni R, Garberini A, Caputo D, Coppola R. Solitary splenic metastasis of ovarian carcinoma: report of two cases. Surg Today. 2008;38(12):1144-1147.

12. Peters AM. Why the spleen is a very rare site for metastases from epithelial cancers. Med Hypotheses. 2012;78(1):26-28.

13. Pujade-Lauraine E, Fujiwara K, Dychter SS, Devgan G, Monk BJ. Avelumab (anti-PD-L1) in platinum-resistant/refractory ovarian cancer: JAVELIN Ovarian 200 Phase III study design. Future Oncol. 2018;14(21):2103-2113.

14. Rose PG, Piver MS, TsukadaY, Lau TS. Metastatic patterns in histologic variants of ovarian cancer. An autopsy study. Cancer. 1989;64(7):1508-1513.

15. Lv M, Li Y, Luo C, Liu P, Yang J. Splenic metastasis of ovarian clear cell adenocarcinoma: a case report and review of the literature. Exp Ther Med. 2014;7(4):982-986.

16. Compérat E, Bardier-Dupas A, Camparo P, Capron F, Charlotte F. Splenic metastases: clinicopathologic presentation, differential diagnosis, and pathogenesis. Arch Pathol Lab Med. 2007;131(6):965-969.

17. Manci N, Bellati F, Muzii L, et al. Splenectomy during secondary cytoreduction for ovarian cancer disease recurrence: surgical and survival data. Ann Surg Oncol. 2006;13(12):1717-1723.

18. Nicklin J, Copeland L, O'Toole R, Lewandowski G, Vaccarello L, Havenar L. Splenectomy as part of cytoreductive surgery for ovarian carcinoma. Int J Gynaecol Obstet. 1996;52(3):333-333.

19. Bacalbasa N, Balescu I, Dima S, Brasoveanu V, Popescu I. Hematogenous splenic metastases as an independent negative prognosis factor at the moment of primary cytoreduction in advanced stage epithelial ovarian cancer: a single center experience. Anticancer Res. 2015;35(10):5649-5654.

20. Ayhan A, Al RA, Baykal C, Demirtas E, Ayhan A, Yüce K. The influence of splenic metastases on survival in FIGO stage IIIC epithelial ovarian cancer. Int J Gynecol Cancer. 2004;14(1):51-56.

21. Hanprasertpong J, Fujiwara K. Splenectomy and surgical cytoreduction in epithelial ovarian cancer: a review. Eur $J$ Cancer Care. 2011;20(3):287-293.

22. Miller EM, Tymon-Rosario J, Xie X, et al. Utilization of intraperitoneal chemotherapy for optimally cytoreduced advanced stage epithelial ovarian cancer: a 10-year single institution experience with a racially diverse urban population. Gynecol Oncol. 2017;147(1):36-40.

23. Alberts DS, Liu PY, Hannigan EV, et al. Intraperitoneal cisplatin plus intravenous cyclophosphamide versus intravenous cisplatin plus intravenous cyclophosphamide for stage III ovarian cancer. $N$ Engl J Med. 1996;335(26):1950-1955.

24. Markman M, Bundy BN, Alberts DS, et al. Phase III trial of standard-dose intravenous cisplatin plus paclitaxel versus moderately high-dose carboplatin followed by intravenous paclitaxel and intraperitoneal cisplatin in small-volume stage III ovarian carcinoma: an intergroup study of the Gynecologic Oncology Group, Southwestern Oncology Group, and Eastern Cooperative Oncology Group. J Clin Oncol. 2001;19(4):1001-1007.

25. Armstrong DK, Bundy B, Wenzel L, et al. Intraperitoneal cisplatin and paclitaxel in ovarian cancer. $N$ Engl J Med. 2006;354(1):34-43.

26. Lee J, Curtin JP, Muggia FM, Pothuri B, Boyd LR, Blank SV. Timing is everything: intraperitoneal chemotherapy after primary or interval debulking surgery for advanced ovarian cancer. Cancer Chemother Pharmacol. 2018;82(1):55-63.

27. Wright JD, Hou JY, Burke WM, et al. Utilization and toxicity of alternative delivery methods of adjuvant chemotherapy for ovarian cancer. Obstet Gynecol. 2016;127(6):985-991.

28. Havrilesky LJ, Alvarez SecordA, Ehrisman JA, et al. Patient preferences in advanced or recurrent ovarian cancer. Cancer. 2014;120(23):3651-3659.
29. Fagotti A, Ferrandina G, Fanfani F, et al. Prospective validation of a laparoscopic predictive model for optimal cytoreduction in advanced ovarian carcinoma. Am J Obstet Gynecol. 2008;199(6):e641-646:642-642.e6.

30. Weitz J, Jaques DP, Brennan M, Karpeh M. Association of splenectomy with postoperative complications in patients with proximal gastric and gastroesophageal junction cancer. Ann Surg Oncol. 2004;11(7):682-689.

31. Chua TC, Yan TD, Saxena A, Morris DL. Should the treatment of peritoneal carcinomatosis by cytoreductive surgery and hyperthermic intraperitoneal chemotherapy still be regarded as a highly morbid procedure? A systematic review of morbidity and mortality. Ann Surg. 2009;249(6):900-907.

32. Eaton MA, Valentine J, Jackson MR, Modrall G, Clagett P. Incidental splenic injury during abdominal vascular surgery: a case-controlled analysis. J Am Coll Surg. 2000;190(1):58-64.

33. Mettke R, Schmidt A, Wolff S, et al. Spleen injuries during colorectal carcinoma surgery. Effect on the early postoperative result. Der Chirurg; Zeitschrift fur alle Gebiete der operativen Medizen. 2012;83(9):809-814.

34. Bisharat N, Omari H, Lavi I, Raz R. Risk of infection and death among post-splenectomy patients. J Infect. 2001;43(3):182-186.

35. Dagbert F, Thievenaz R, Decullier E, et al. Splenectomy increases postoperative complications following cytoreductive surgery and hyperthermic intraperitoneal chemotherapy. Ann Surg Oncol. 2016;23(6): 1980-1985.

36. Magtibay PM, Adams PB, Silverman MB, Cha SS, Podratz KC. Splenectomy as part of cytoreductive surgery in ovarian cancer. Gynecol Oncol. 2006;102(2):369-374.

37. Eisenkop SM, Spirtos NM, Lin W-CM. Splenectomy in the context of primary cytoreductive operations for advanced epithelial ovarian cancer. Gynecol Oncol. 2006;100(2):344-348.

38. Cadili A, de Gara C. Complications of splenectomy. Am J Med. 2008;121(5):371-375.

39. Waghorn DJ. Overwhelming infection in asplenic patients: current best practice preventive measures are not being followed. J Clin Pathol. 2001;54(3):214-218.

40. Buzelé R, Barbier L, Sauvanet A, Fantin B. Medical complications following splenectomy. J Visc Surg. 2016;153(4):277-286.

41. Spencer NJB, Spencer JA, Perren TJ, Lane G. CT appearances and prognostic significance of splenic metastasis in ovarian cancer. Clin Radiol. 1998;53(6):417-421.

42. Piura E, Piura B. Splenic metastases from female genital tract malignancies]. Harefuah. 2010;149(5):315-320.

43. Kugimiya N, Suto R, Kaneda Y, et al. A case of solitary splenic metastasis from ovarian cancer resected by laparoscopic partial splenectomy. Jpn J Gastroenterol Surg. 2008;41(2):224-228.

44. Marriqoun-Harris F, Rhou YJJ, Storey D, Pather S. Prolonged survival after laparoscopic splenectomy for recurrent ovarian cancer and no adjuvant therapy: a report and review of the literature. SAfr J Gynaecol Oncol. 2013;5(2):58-61.

45. Mccann CK, Growdon WB, Munro EG, et al. Prognostic significance of splenectomy as part of initial cytoreductive surgery in ovarian cancer. Ann Surg Oncol. 2011;18(10):2912-2918.

46. Nicklin JL, Copeland LJ, O’Toole RV, Lewandowski GS, Vaccarello L, Havenar LP. Splenectomy as part of cytoreductive surgery for ovarian carcinoma. Gynecol Oncol. 1995;58(2):244-247.

47. Chen L-May, Leuchter RS, Lagasse LD, Karlan BY. Splenectomy and surgical cytoreduction for ovarian cancer. Gynecol Oncol. 2000;77(3):362-368.

48. Zapardiel I, Peiretti M, Zanagnolo V, et al. Splenectomy as part of primary cytoreductive surgery for advanced ovarian cancer: a retrospective cohort study. Int J Gynecol Cancer. 2012;22(6):968-973. 


\section{Publish your work in this journal}

Cancer Management and Research is an international, peer-reviewed open access journal focusing on cancer research and the optimal use of preventative and integrated treatment interventions to achieve improved outcomes, enhanced survival and quality of life for the cancer patient. The manuscript management system is completely online and includes a very quick and fair peer-review system, which is all easy to use. Visit http://www.dovepress.com/testimonials.php to read real quotes from published authors.

Submit your manuscript here: https://www.dovepress.com/cancer-management-and-research-journal 